\title{
On the Use of Recursive Evaluation of Derivatives and Padé Approximation to Solve the Blasius Problem
}

\author{
Asai Asaithambi \\ School of Computing, University of North Florida, Jacksonville, FL 32224, USA \\ Correspondence should be addressed to Asai Asaithambi; asai.asaithambi@gmail.com
}

Received 25 October 2015; Accepted 30 December 2015

Academic Editor: Mikhail Tokar

Copyright (C) 2016 Asai Asaithambi. This is an open access article distributed under the Creative Commons Attribution License, which permits unrestricted use, distribution, and reproduction in any medium, provided the original work is properly cited.

The Blasius problem is one of the well-known problems in fluid mechanics in the study of boundary layers. It is described by a third-order ordinary differential equation derived from the Navier-Stokes equation by a similarity transformation. Crocco and Wang independently transformed this third-order problem further into a second-order differential equation. Classical series solutions and their Padé approximants have been computed. These solutions however require extensive algebraic manipulations and significant computational effort. In this paper, we present a computational approach using algorithmic differentiation to obtain these series solutions. Our work produces results superior to those reported previously. Additionally, using increased precision in our calculations, we have been able to extend the usefulness of the method beyond limits where previous methods have failed.

\section{Introduction}

The boundary value problem described by

$$
\begin{aligned}
f^{\prime \prime \prime}(\eta)+\beta_{0} f(\eta) f^{\prime \prime}(\eta) & =0, \\
f(0) & =f^{\prime}(0)=0, \\
f^{\prime}(\infty) & =1
\end{aligned}
$$

is called the Blasius problem [1]. It is one of the well-known problems in fluid mechanics in the study of boundary layers.

Blasius [1], Howarth [2], and Asaithambi [3] provide direct analytical and numerical treatments of (1). For instance, with $\beta_{0}=1 / 2$, Blasius [1] obtained the series solution

$$
f(\eta)=\sum_{j=0}^{\infty}\left(-\frac{1}{2}\right)^{j} \frac{A_{j} \alpha^{j+1}}{(3 j+2) !} \eta^{3 j+2},
$$

with

$$
\begin{aligned}
& A_{0}=A_{1}=1, \\
& A_{j}=\sum_{r=0}^{j-1}\left(\begin{array}{c}
3 j-1 \\
3 r
\end{array}\right) A_{r} A_{j-r-1}, \quad j \geq 2,
\end{aligned}
$$

and $\alpha$ representing the unknown $f^{\prime \prime}(0)$. For this case $\left(\beta_{0}=\right.$ $1 / 2)$, Howarth [2] computed the numerical value $f^{\prime \prime}(0) \approx$ 0.33206. Liao [4] takes the approach of the Homotopy Analysis Method (HAM) and obtains "an explicit, totally analytic approximate solution" for the Blasius problem with $\beta_{0}=1 / 2$.

Asaithambi [3] solved numerically the problem corresponding to $\beta_{0}=1$ using Taylor series and shooting and obtained $f^{\prime \prime}(0) \approx 0.469600$. Using suitable substitutions, Fang et al. [5] have shown that it suffices to consider the case $\beta_{0}=1$. Using Fang et al. [5], it is easy to see that the value $\left.f^{\prime \prime}(0)\right|_{\beta_{0}=1}=0.469600$ corresponds to $\left.f^{\prime \prime}(0)\right|_{\beta_{0}=1 / 2}=$ $\left.f^{\prime \prime}(0)\right|_{\beta_{0}=1} / \sqrt{2}$. Thus the numerical value corresponding to Asaithambi [3] will be $0.469600 / \sqrt{2}=0.332057$ which is in agreement with Howarth [2]. Thus, for the rest of this paper, we will consider only the case corresponding to $\beta_{0}=1$.

Using the transformation

$$
\begin{aligned}
& x=f^{\prime}(\eta), \\
& y=f^{\prime \prime}(\eta),
\end{aligned}
$$

Wang [6] reduced the problem (1) (with $\beta_{0}=1$ ) to

$$
\frac{d^{2} y}{d x^{2}}+\frac{x}{y}=0, \quad 0 \leq x<1,
$$


subject to the boundary conditions

$$
\begin{aligned}
\frac{d y}{d x}(0) & =0, \\
y(0) & =f^{\prime \prime}(0), \\
y(1) & =0 .
\end{aligned}
$$

It has been recently reported [7, 8] that Crocco [9] had used transformation (4) already in the 1940s. Since no closed-form solutions are available in general, the search for efficient series and numerical solutions to (5)-(6) has been an active research topic.

Ahmad [7] obtains a series solution for (5) as

$$
y(x)=\sum_{j=0}^{\infty} a_{j} x^{j}
$$

where $a_{j}$ 's satisfy

$$
\begin{aligned}
& 3 j(3 j-1) a_{3 j} \\
& =-\frac{1}{\alpha} \sum_{r=1}^{j-1}(3 j-3 r)(3 j-3 r-1) a_{3 r} a_{3 j-3 r},
\end{aligned}
$$

with $a_{0}=\alpha, a_{3}=-1 / 6 \alpha$, and $\alpha=f^{\prime \prime}(0)$. Expanded through the first few terms, this solution looks like

$$
\begin{aligned}
y(x) \approx & \alpha-\frac{1}{6 \alpha} x^{3}-\frac{1}{180 \alpha^{3}} x^{6}-\frac{1}{2160 \alpha^{5}} x^{9} \\
& -\frac{1}{19008 \alpha^{7}} x^{12}-\cdots
\end{aligned}
$$

This series appears to be identical to the one obtained by Wang [6] who used Adomian decomposition. All of these researchers who obtained the series in terms of $\alpha=f^{\prime \prime}(0)$ then proceeded to solve for $\alpha$ by satisfying the boundary condition $y(1)=0$. This, in essence, is the approach known as shooting in numerical analysis.

Ahmad [7] obtains an increasing sequence of approximations to $\alpha$ and a decreasing sequence and concludes that $\alpha$ satisfies $0.469597<\alpha<0.4696064$. Hashim [10] and Ahmad and Albarakati [11] use a Padé approximation to improve upon the results of Wang [6] and Ahmad [7], respectively, but report that as the number of terms in the series solution increases, the accuracy of the $\alpha$ produced by the corresponding Padé approximation suffers.

In this paper, we develop a shooting method that successfully obtains Taylor series expansions of arbitrarily large orders by computing exact derivatives, not approximations to the derivatives, directly by using recursive formulas derived from the differential equation itself. Also, our approach does not require the use of symbolic manipulation packages as it does not involve extensive algebraic manipulations. Finally, by using increased precision, we are able to obtain superior Padé approximants to the solution as well.

\section{Method of Solution}

As with any shooting method, we convert the two-point boundary-value problem to an initial-value problem and determine an appropriate set of boundary conditions at one end so that the boundary condition at the other end is satisfied. To be specific, we will solve (5)-(6) by supplying the initial value $(d y / d x)(0)=0$ and a suitable value for $y(0)=$ $f^{\prime \prime}(0)$ to obtain a solution $y(x)$ for which the boundary condition $y(1)=0$ in (6) is satisfied.

Accordingly, we begin by letting $y(0)=\alpha$ and obtain the solution of (5)-(6) as Taylor series expansion of degree $J$. In other words, we first solve the initial value problem

$$
\frac{d^{2} y}{d x^{2}}+\frac{x}{y}=0, \quad 0<x<1,
$$

subject to the conditions

$$
\begin{aligned}
\frac{d y}{d x}(0) & =0, \\
y(0) & =\alpha .
\end{aligned}
$$

In order to indicate its dependence on both $x$ and $\alpha$, we denote the solution thus obtained as $y_{J}(x ; \alpha)$. Now, if we let

$$
p(\alpha)=y_{J}(1 ; \alpha)
$$

then we wish to obtain $\alpha$ for which $p(\alpha)=0$.

Our method will begin by obtaining the $J$ th-order Taylor series solution of (10)-(11) in the form

$$
y(x)=\sum_{j=0}^{J}(y)_{j} x^{j},
$$

in which the notation

$$
(y)_{j}=\frac{1}{j !} \frac{d^{j} y}{d x^{j}}(0),
$$

has been used. The quantity $(y)_{j}$ is called the $j$ th Taylor coefficient for $y(x)$ around $x=0$. Thus, we wish to obtain the Taylor coefficients $(y)_{j}$ for $j=1, \ldots, J$ for arbitrary $J$.

For this purpose in relation to this problem, we will use the following formulas.

(1) For two functions $r(x)$ and $s(x)$ satisfying $d r / d x=s$,

$$
(r)_{j+1}=\frac{1}{j+1}(s)_{j} .
$$

(2) For any two functions $r(x)$ and $s(x)$,

$$
\left(\frac{r}{s}\right)_{j}=\left(\frac{1}{(s)_{0}}\right)\left\{(r)_{j}-\sum_{i=1}^{j}(s)_{i}\left(\frac{r}{s}\right)_{j-i}\right\} .
$$

The formulas (15)-(16) are examples of algorithmic/automatic differentiation formulas, which may be used to evaluate successive Taylor coefficients recursively without deriving 
symbolic expressions for the corresponding derivatives. Note that these formulas evaluate the exact derivatives and are not comparable to any finite-difference formulas (numerical), nor are they formulas that require the use of symbolic manipulation systems. A more extensive yet preliminary treatment of automatic differentiation (recursive evaluation of Taylor coefficients) may be found in Moore [12] and other references cited there.

2.1. Evaluation of Taylor Coefficients. We rewrite (10)-(11) as an equivalent system in the form

$$
\begin{gathered}
\frac{d y}{d x}=u, \\
y(0)=\alpha, \\
\frac{d u}{d x}=-\frac{x}{y}, \\
u(0)=0 .
\end{gathered}
$$

Using (15)-(16) in (17) yields, for $j \geq 0$,

$$
\begin{aligned}
(y)_{j+1} & =\frac{1}{j+1}(u)_{j}, \\
\left(T_{1}\right)_{j} & =-\left(\frac{1}{(y)_{0}}\right)\left\{(x)_{j}-\sum_{i=1}^{j}(y)_{i}\left(T_{1}\right)_{j-i}\right\}, \\
(u)_{j+1} & =\frac{1}{j+1}\left(T_{1}\right)_{j} .
\end{aligned}
$$

In (18), we have introduced a temporary variable $T_{1}$ to make the presentation of the calculation easier to understand. The initial conditions $y(0)=\alpha$ and $u(0)=0$ in (17) now take the form

$$
\begin{aligned}
& (y)_{0}=\alpha, \\
& (u)_{0}=0 .
\end{aligned}
$$

Also, note that the Taylor coefficients $(x)_{j}$ for the function $x$ are given by

$$
(x)_{j}= \begin{cases}x, & \text { if } j=0 \\ 1, & \text { if } j=1 \\ 0, & \text { otherwise }\end{cases}
$$

Therefore, with an initial "guess" for $\alpha$, it is possible to compute the numerical values of $(y)_{j}$ and $(u)_{j}$ for arbitrary $J$ recursively. A quick examination of the calculations for the present problem shows that $(y)_{3 j+1}=(y)_{3 j+2}=0$ for $j \geq 0$. In other words, the only nonzero terms in the Taylor series are those that correspond to powers of $x$ that are multiples of 3 (the constant term $\alpha$, the $x^{3}$ term, the $x^{6}$ term, etc.).

2.2. Removing the Dependence on the Initial Guess for $\alpha$. We realized that it will be useful to explicitly separate the dependence of these coefficients on $\alpha$ so that the series we obtain may be compared with those obtained previously. As we proceeded to do this, we discovered an alternate approach to solving for $\alpha$.

As a clarification for this, let us rewrite (9) as

$$
y(x)=\alpha+\sum_{j=1}^{\infty} \frac{\delta_{j}}{\alpha^{2 j-1}} x^{3 j} .
$$

Comparing (21) with (9) term by term yields, for instance,

$$
\begin{aligned}
& \delta_{1}=-\frac{1}{6}, \\
& \delta_{2}=-\frac{1}{180}, \\
& \delta_{3}=-\frac{1}{2160}, \\
& \delta_{4}=-\frac{1}{19008}, \quad \text { etc. }
\end{aligned}
$$

Next, comparing (21) with (13) yields,

$$
\delta_{j}=(y)_{3 j} \alpha^{2 j-1} .
$$

We were able to verify this by running the calculations with initial guesses for $\alpha=0.3$ and 0.5 . In both cases, we obtained the same values for the $\delta_{j}$, as in (22).

2.3. Solving for $\alpha$. Suppose we obtain Taylor coefficients of orders through $J$; then we can compute $\delta_{j}$ for $j=$ $1,2, \ldots,\lfloor J / 3\rfloor$ using (23). In order to impose the condition $y(1)=0$, we truncate the series in (21) after $j=\lfloor J / 3\rfloor$, set $x=1$, and set the resulting summation to zero. We obtain

$$
\alpha+\sum_{j=1}^{\lfloor J / 3\rfloor} \frac{\delta_{j}}{\alpha^{2 j-1}}=0
$$

Dividing throughout by $\alpha$, setting $\gamma=1 / \alpha^{2}$, defining $\delta_{0}=$ 1 in (24), and denoting the resulting polynomial as $g(\gamma)$, we obtain

$$
g(\gamma)=\sum_{j=0}^{\lfloor J / 3\rfloor} \delta_{j} \gamma^{j}=0
$$

Since $g(\gamma)=0$ in (25) is a polynomial equation, it can be solved easily using Newton's method.

Alternatively, with $2 L=\lfloor J / 3\rfloor$, we can obtain a $[L / L]$ Padé approximant for $g(\gamma)$ as

$$
g_{[L / L]}(\gamma)=\frac{p(\gamma)}{q(\gamma)}=\frac{p_{0}+p_{1} \gamma+\cdots+p_{L} \gamma^{L}}{1+q_{1} \gamma+q_{2} \gamma^{2}+\cdots+q_{L} \gamma^{L}}
$$

and then solve $p(\gamma)=0$ instead. Standard numerical algorithms are available [13] for obtaining the Padé approximant (26), and they involve the solution of linear systems of order $L$. 
TABLE 1: Taylor series solutions $\left(\beta_{0}=1\right)$ compared (increasing sequence).

\begin{tabular}{lccc}
\hline$J$ & Present & $k$ & Ahmad [7] \\
\hline 3 & 0.408248290 & 1 & 0.408248 \\
6 & 0.441742835 & 2 & 0.441743 \\
15 & 0.460566286 & 5 & 0.460566 \\
21 & 0.463662320 & 7 & 0.463662 \\
45 & 0.467293212 & 15 & 0.467293 \\
75 & 0.468366120 & 25 & 0.468366 \\
150 & 0.469065604 & 50 & 0.469066 \\
300 & 0.469365358 & 100 & 0.469365 \\
600 & 0.469495691 & 200 & 0.469496 \\
900 & 0.469534749 & 300 & 0.469535 \\
3000 & 0.469583484 & 1000 & 0.469583 \\
6000 & 0.469592426 & 2000 & 0.469592 \\
9000 & 0.469595183 & 3000 & 0.469595 \\
12000 & 0.469596501 & 4000 & 0.469597 \\
\hline
\end{tabular}

Once the root $\gamma^{*}$ for which $g\left(\gamma^{*}\right)=0$ or $p\left(\gamma^{*}\right)=0$ is obtained, then the value of $\alpha$ is computed as $\alpha^{*}=1 / \sqrt{\gamma^{*}}$.

This approach is the same as Ahmad and Albarakati's [11]. However, as our experimentation with arbitrarily large orders $J$ has revealed, our results are much superior and extend far beyond the limits encountered by [11] where their method failed to produce results.

\section{Numerical Results and Discussion}

In this section, we present our results and compare them with those obtained by Ahmad [7] and by Ahmad and Albarakati [11]. It must be noted that the index values these authors have used are not the same as the index $J$ we have used for the order of the Taylor series, but they are closely related. In order to make things clearer, we will tabulate the index $J$ of our method corresponding to the indexes used in the previous works.

Ahmad [7] computes an increasing sequence converging to the desired value of $\alpha$ and then a decreasing sequence also converging to $\alpha$ and concludes that $\alpha$ satisfies $0.469597<\alpha<$ 0.4696064 . The index $k$ used in [7] satisfies $J=3 k$, where $J$ is the order of the Taylor series obtained by our method. For instance, when $k=2$ is used in [7], the corresponding Taylor series is of order 6 . Table 1 shown indicates that our results for $\alpha$ obtained by solving $g(\gamma)=0$ are in excellent agreement with the increasing sequence of [7].

For all computations in Table 1 we have used an initial value of $\alpha=0.5$ to calculate the Taylor coefficients. As we pointed out earlier, this initial value will not impact the values obtained for the $\delta_{j}$ values. However, for the solution of $g(\gamma)=$ 0 , we need an initial guess for $\gamma$. We have used $\gamma=4$, which corresponds to $1 / \alpha^{2}$ with $\alpha=0.5$. Our computations yielded the same results as those of [7] for $k$ values through 300, when double precision arithmetic was used. For $k \geq 1000$, we were able to obtain the results reported using quadruple precision.
TABLE 2: Padé approximants compared.

\begin{tabular}{lccc}
\hline$J$ & Present & $i$ & Ahmad and Albarakati [11] \\
\hline 12 & 0.463256776 & 2 & 0.463257 \\
24 & 0.468060891 & 4 & 0.468061 \\
36 & 0.468956035 & 6 & 0.468956 \\
48 & 0.469256787 & 8 & 0.468997 \\
60 & 0.469390186 & 10 & 0.469025 \\
72 & 0.469459891 & 12 & 0.469051 \\
84 & 0.469500493 & 14 & 0.469075 \\
96 & 0.469526045 & 16 & 0.469097 \\
108 & 0.469543087 & 18 & 0.469118 \\
120 & 0.469554976 & 20 & 0.469124 \\
132 & 0.469563577 & 22 & 0.469977 \\
138 & 0.469567005 & 23 & 0.474672 \\
144 & 0.469569990 & 24 & Unavailable \\
\hline
\end{tabular}

TABLE 3: Padé approximants for larger values of $J$.

\begin{tabular}{lc}
\hline$J$ & $\alpha$ \\
\hline 120 & 0.46955497638 \\
480 & 0.46959296614 \\
1920 & 0.46959844847 \\
7680 & 0.46959964975 \\
\hline
\end{tabular}

Next, we compare our Padé approximant results with the corresponding results of Ahmad and Albarakati [11]. Once again, the index $i$ used in the paper by Ahmad and Albarakati [11] corresponds to retaining through the $x^{6 i}$ term in the Taylor series expansion. In other words, $i$ satisfies $J=6 i$, where $J$ is the order of Taylor cries we have used. For instance, when $i=2$ is used in [11], the corresponding Taylor series used to obtain the Padé approximant is actually of order 12 . The Padé approximant yields a value of $\alpha$, denoted as $\alpha^{i}$ in [11]. Table 2 shows the $\alpha$ values obtained by solving $p(\gamma)=0$ in our notation, with the corresponding $\alpha^{i}$ values reported in [11] for values of $J$ not exceeding 138. As is evident from Table 2, our results are superior in accuracy compared to the results of [11], beginning with the case corresponding to their $i=8$ (or our $J=48$ ).

Also, we were able to continue our calculations much beyond the limiting case observed of $i=24$ by [11]. In Table 3 , we present results we obtained for larger values of $J$. As the $J$ value is increased, by supplying the final $\alpha$ values obtained for smaller values of $J$ as initial guesses for larger $J$, we were able to carry out the calculation of Padé approximants through $J=7680$ (or $i=1280$ ) with no difficulty, and we obtain $\alpha \approx 0.46959964975$.

It is important to realize that as the order of the Taylor series increases, the order of the linear system to be solved to obtain the coefficients in the Padé approximant will also increase. As these linear systems are known to have coefficient matrices which are close to being singular, it is necessary to carry out the calculations in higher precision and use iterative refinement of the solution obtained as well. We have 
used quadruple precision in all our calculations of the Padé approximants reported in Table 3.

We close our discussion by comparing our approach with the HAM approach of Liao [4], even though their goal was to obtain an analytic approximate solution, while we have obtained numerical solution by Taylor series. The present result of $\left.f^{\prime \prime}(0)\right|_{\beta_{0}=1} \approx 0.469599$ (from Table 3) corresponds to the result of $\left.f^{\prime \prime}(0)\right|_{\beta_{0}=1 / 2} \approx 0.332056$ on using the observation by Fang et al. [5]. This result is in agreement with Liao [4]. It is important to realize that the computational complexity of the two techniques cannot be directly compared because a $k$ th order Taylor solution obtained by the present method and a $k$ th-order HAM solution obtained by Liao [4] are entirely different. The method of Liao [4] is a technique for obtaining explicit, analytical approximate solutions for general nonlinear problems, which has been applied to the Blasius problem with $\beta_{0}=1 / 2$. Liao's [4] technique obtains a series solution that uses two parameters $\beta$ and $\hbar$ and two embedding functions $A(p)$ and $B(p)$, studies the mathematical structure of the series by using symbolic manipulation packages such as MATHEMATICA, and derives recursive formulas for the coefficients of a series involving exponential functions, which need to be evaluated for each term in the HAM solution. The method is applied directly on the Blasius problem (1) involving the independent variable $\eta$. However, because of the goal of obtaining analytical solutions in the HAM technique, obtaining the underlying symbol manipulations and the recursive formulas may involve a significant amount of analytical manipulations before calculations may be carried out. Additionally, the nature of the analytical manipulations and the convergence will depend on the choices of the parameters and the embedding functions. On the other hand, note that the present method is a numerical method that obtains a Taylor series solution of the transformed Blasius problem (4), does not rely on the use of symbol manipulation packages, obtains the coefficients in a series solution in a recursive manner to construct Padé approximants, and does not require repeated calculation of exponential or any other nonlinear functions. However, the present method does require the solution of large systems of linear equations in extended precision as the order of the desired Padé approximant is increased. The simplicity and overall performance of the present method are well worth the effort expended in the solution of the linear systems.

\section{Conclusions}

We have developed a computational method for obtaining arbitrarily larger order Taylor series solutions of the Blasius problem by evaluating exact derivatives for the coefficients in the series using algorithmic differentiation. From the series solutions thus obtained, we also computed (diagonal) Padé approximants. Our method does not use symbol manipulation packages or difference formulas for calculating the derivatives needed in the Taylor series. Quadruple precision arithmetic and iterative refinement were used in the calculations related to obtaining Padé approximants. The results obtained by our present method are superior to those obtained previously and are extensible beyond the limits where previous methods have failed.

\section{Conflict of Interests}

The author declares that there is no conflict of interests regarding the publication of this paper.

\section{References}

[1] H. Blasius, "Grenschhichten in Flussigkeiten miy kleiner Reibung," Zeitschrift für angewandte Mathematik und Physik, vol. 56, pp. 1-37, 1908.

[2] L. Howarth, "On the solution of the laminar boundary layer equations," Proceedings of the London Mathematical Society A, vol. 164, pp. 547-579, 1938.

[3] A. Asaithambi, "Solution of the Falkner-Skan equation by recursive evaluation of Taylor coefficients," Journal of Computational and Applied Mathematics, vol. 176, pp. 203-214, 2005.

[4] S. J. Liao, "An explicit, totally analytic approximate solution for Blasius' viscous flow problems," International Journal of NonLinear Mechanics, vol. 34, pp. 759-778, 1999.

[5] T. Fang, F. Guo, and C. F. Lee, "A note on the extended Blasius equation," Applied Mathematics Letters, vol. 19, pp. 613-617, 2006.

[6] L. Wang, "A new algorithm for solving classical Blasius equation," Applied Mathematics and Computation, vol. 157, pp. 1-9, 2004.

[7] F. Ahmad, "Application of Crocco-Wang equation to the Blasius problem," Electronic Journal: Technical Acoustics, vol. 2, 2007.

[8] C.-S. Yih, Fluid Mechanics-A Concise Introduction to the Theory, McGraw-Hill, New York, NY, USA, 1969.

[9] L. Crocco, "Sull strato limite laminare nei gas lungo una lamina plana," Rendiconti di Matematica e delle Sue Applicazioni Serie 5, vol. 21, pp. 138-152, 1941.

[10] I. Hashim, "Comments on 'A new algorithm for solving classical Blasius equation' by L. Wang," Applied Mathematics and Computation, vol. 176, pp. 700-703, 2006.

[11] F. Ahmad and W. A. Albarakati, "Application of Padé approximation to the Blasius problem," Proceedings of the Pakistan Academy of Sciences, vol. 44, pp. 17-19, 2007.

[12] R. E. Moore, Methods and Applications of Interval Analysis, SIAM Publications, Philadelphia, Pa, USA, 1979.

[13] N. S. Asaithambi, Numerical Analysis: Theory and Practice, Saunders College Publishing, 1995. 

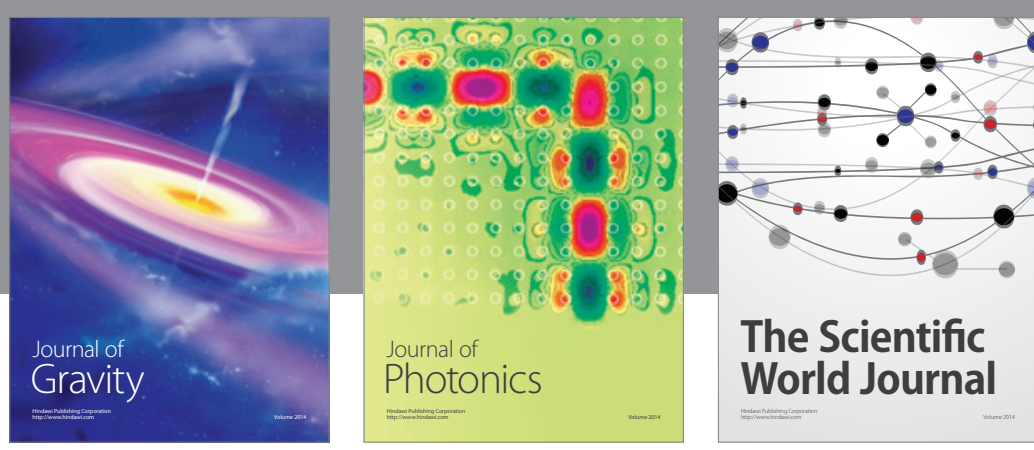

The Scientific World Journal
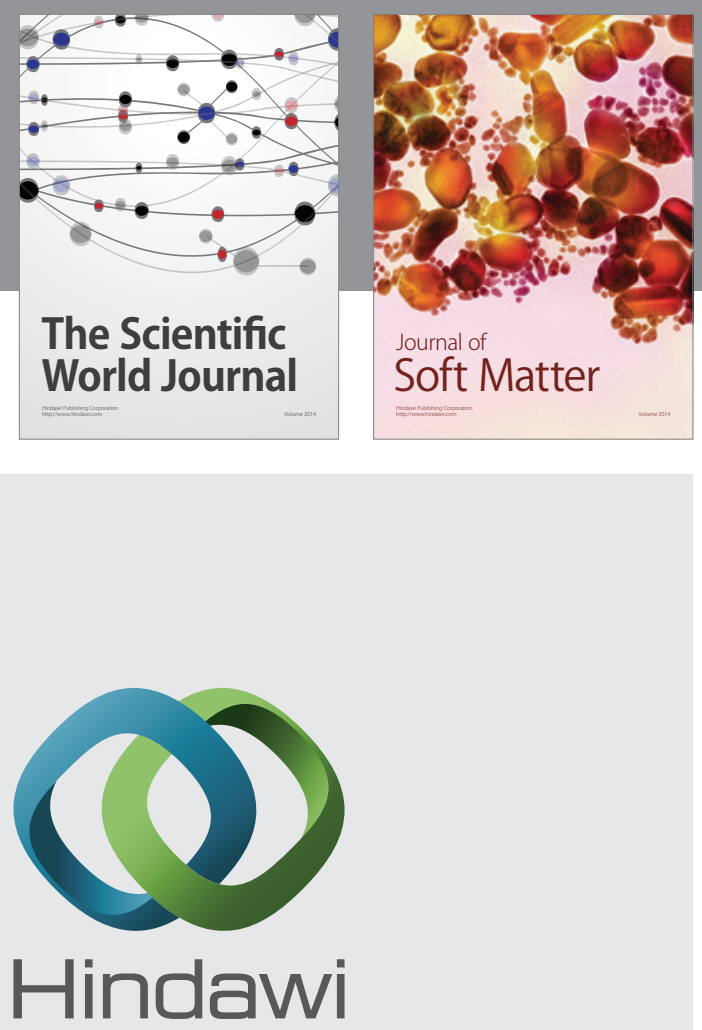

Submit your manuscripts at

http://www.hindawi.com

nternational Journal of

Statistical Mechanics
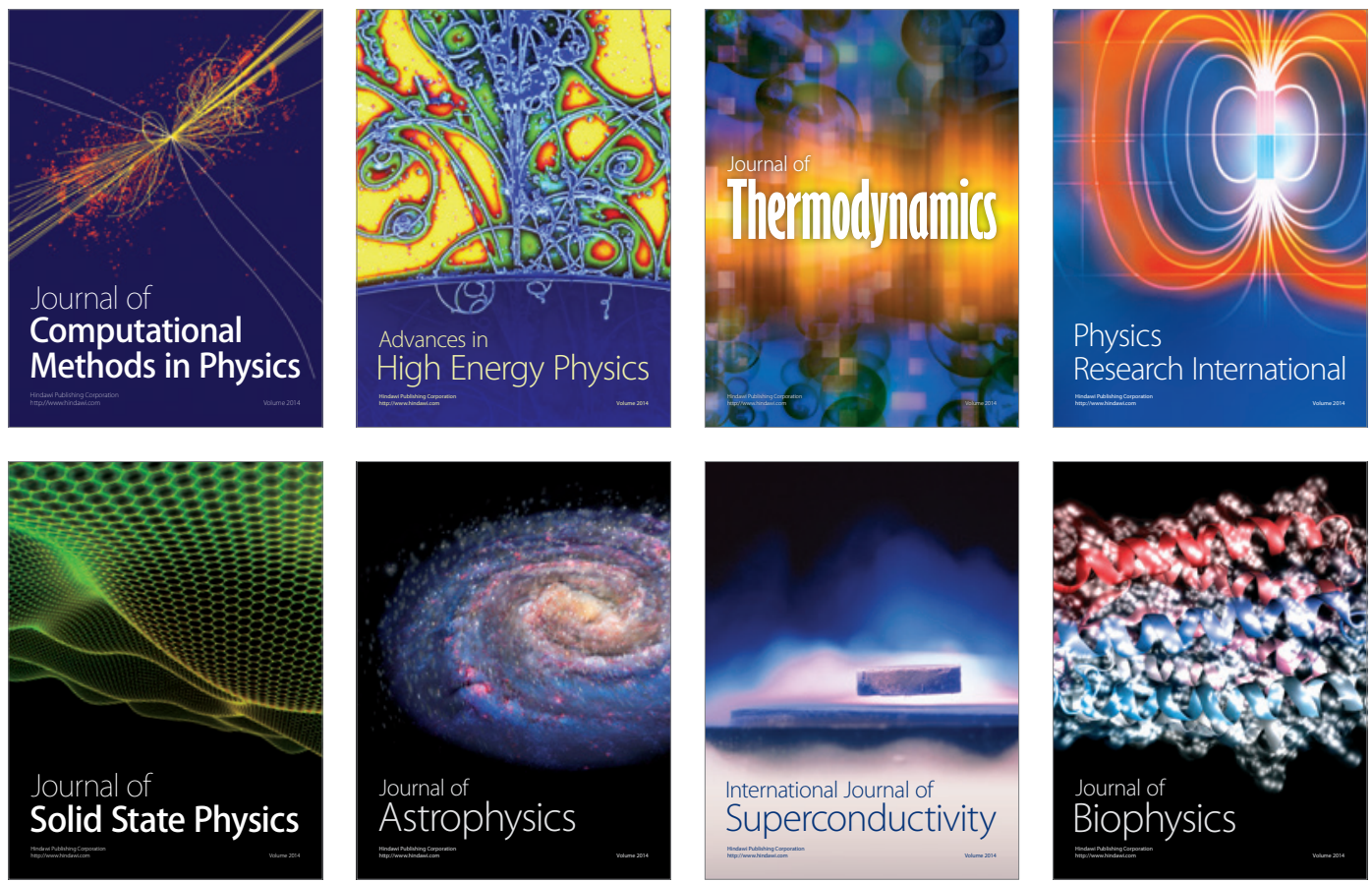
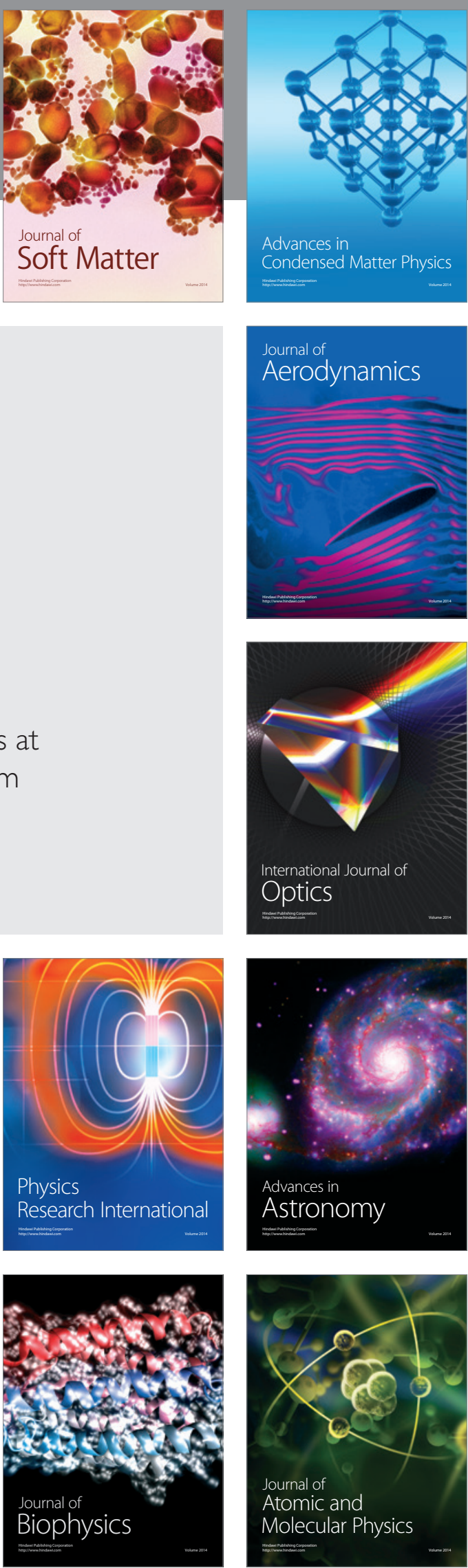